\title{
Bibliographie France Daigle (2003-2017)
}

Benoit Doyon-Gosselin (en collaboration avec Anahita Shafie)

Note : En 2004, dans le cadre d'un numéro de la revue Voix et images portant sur l'écrivaine acadienne France Daigle, j'avais préparé une première bibliographie scientifique de l'auteure. Elle comprenait tout ce que France Daigle avait publié et tout ce qui avait été publié sur la romancière de 1983 à 2003. La présente bibliographie scientifique constitue la suite. Elle ne reprend aucun des éléments de la bibliographie de 2004, mais inclut plutôt toutes les œuvres publiées de France Daigle et tous les travaux sur l'auteure parus entre 2003 et 2017.

\section{Euvres}

\section{I.1. Roman}

Pour sûr. Montréal : Boréal, 2011.

Sans jamais parler du vent. Roman de crainte et d'espoir que la mort arrive à temps. Ed. critique établie par Monika Boehringer. Moncton : Institut d'études acadiennes, 2012.

Pour sûr, $2^{\mathrm{e}}$ éd. Montréal : Boréal, 2013.

Sans jamais parler du vent, Film d'amour et de dépendance, Histoire de la maison qui brûle, $2^{\mathrm{e}}$ éd. Sudbury : Prise de parole, 2013.

1953. Chronique d'une naissance annoncée, $2^{\mathrm{e}}$ éd. Sudbury : Prise de parole, 2014.

Variations en B et K, La beauté de l'affaire, La vraie vie, $2^{\mathrm{e}}$ éd. Sudbury : Prise de parole, 2016.

\section{I.2. Poésie}

Poèmes pour vieux couples. Moncton : Perce-Neige, 2016.

\section{I.3. Publications dans des anthologies ou ouvrages collectifs}

« Sur les traces de Marianne Godbout, cordonnière et savetière », « Méditerranéennes 1 », «Méditerranéennes 2 », « Pour Zahava où qu'elle soit », " Et cela dura », " Le concert », « Le principe de la culture », «Il pleut». Anthologie de la poésie des femmes en Acadie. Dir. Monika Boehringer. Moncton : Perce-Neige, 2014. 131-143.

«What is it that Hurts? ». Granta 141 (automne 2017) : 239-246. 


\section{I.4. Inédits}

\section{I.4.1. Théâtre}

En pelletant de la neige. Moncton : Moncton-Sable, 2003.

Sans jamais parler du vent. Moncton : Moncton-Sable, 2004.

Histoire de la maison qui brûle. Moncton : Moncton-Sable, 2007.

\section{Traduction des romans de France Daigle}

Life's Little Difficulties. Tr. Robert Majzels. Toronto : House of Anansi P, 2004.

For Sure. Tr. Robert Majzels. Toronto : House of Anansi P, 2013.

\section{Prix et distinctions}

Prix du Lieutenant-gouverneur du Nouveau-Brunswick, en 2011, pour l'ensemble de son œuvre.

Prix du Gouverneur général, en 2012, pour le roman Pour sûr.

Prix Antonine-Maillet-Acadie Vie, en 2012, pour le roman Pour sûr.

Prix Champlain, en 2012, pour le roman Pour sûr.

Prix Éloizes, en 2014, pour le roman Pour sûr.

\section{Réception critique et études}

IV.1. Livre

Doyon-Gosselin, Benoit. Pour une herméneutique de l'espace : l'œuvre romanesque de J.R. Léveillé et France Daigle. Québec : Nota Bene, 2012.

\section{IV.2. Articles de fonds et chapitres de livres}

Godin, André. « La parole retrouvée ». Dialogues francophones 8-9 (2003) : 223-231.

Gyurcsik, Margareta. "Une écrivaine acadienne "fin de siècle" : France Daigle ». Dialogues francophones 7 (2003) : 125-132.

Leclerc, Catherine. « Dieppe en voyage chez France Daigle et Robert Majzels : Pas pire en traduction ». Dialogues francophones 8-9 (2003) : 231-244. 
Boudreau, Raoul. «Le rapport à la langue dans les romans de France Daigle : du refoulement à l'ironie ». Voix et Images 87 (printemps 2004) : 31-45.

Boudreau, Raoul. «Le dire de l'inhospitalité comme poétique du roman ». Le dire de l'hospitalité. Dir. Lise Gauvin, Pierre L'Hérault et Alain Montandon. Clermont-Ferrand: PU Blaise Pascal, 2004. 157-166.

Den Toonder, Jeannette. «Dépassement des frontières et ouverture dans Pas pire ». Voix et Images 87 (2004) : 57-68.

Doyon-Gosselin, Benoit et Jean Morency. « Le monde de Moncton, Moncton ville du monde : l'inscription de la ville dans les romans récents de France Daigle ». Voix et Images 87 (2004) : 6983.

Dumontet, Danielle. "France Daigle entre autofiction et fiction autobiographique ». Neue Romania 29 (2004) : 107-125.

Gaudet, Jeannette. «France Daigle et les représentations du moi : une étude de Pas pire (1998) ». Nouvelles études francophones 19.2 (2004) : 157-171.

Gyurcsik, Margareta. «L'hétérogène au pluriel : Naïm Kattan, Réjean Ducharme, France Daigle ». Le Canada sous le signe de la migration et du transculturalisme. Dir. Klaus-Dieter Ertler et Martin Löschnigg. Frankfurt am Main : Lang, 2004. 73-95.

Gyurcsik, Margareta. «Temps d'Acadie, temps de Québec, temps d'Europe ». La neige, la même et autre. Essai sur le roman québécois contemporain. Timisoara : PU de Vest, 2004. 161-182.

Leclerc, Catherine. «L'Acadie rayonne : lire France Daigle à travers sa traduction ». Voix et Images 87 (2004) : 85-100.

Paré, François. « France Daigle. Intermittences du récit ». Voix et Images 87 (2004) : 47-55.

Boehringer, Monika. "Sexual/Textual Politics in "Chronicles of a Death and a Birth Foretold" : 1953 by France Daigle ». Interdisciplinary and Cross-Cultural Narratives, Dir. Mark C. Anderson et Irene Maria F. Blayer. New York : Lang, 2005. 151-161.

Ertler, Klaus-Dieter, Andrea Maria Humpl et Daniela Maly. «France Daigle und die akadische Identität ». Ave Maria Stella. Eine kulturwissenschaftliche Einführung in die Acadie. Frankfurt am Main : Lang, 2005. 164-174.

Gyurcsik, Margareta. «Regards croisés sur 1'Europe et l'Acadie : 1953 de France Daigle ». L'émergence et la reconnaissance des études acadiennes : à la rencontre de soi et de l'autre. Dir. Marie-Linda Lord. Moncton : Association internationale des études acadiennes, 2005. 56-70.

Leclerc, Catherine. «Between French and English, Between Ethnography and Assimilation : Strategies for Translating Moncton's Acadian Vernacular ». TTR : Traduction, Terminologie, Rédaction 18.2 (2005) : 161-192. 
Modenesi, Marco. «L'Acadie par transparence. Les romans de France Daigle ». Culture et letterature di lingua francese in Canada. Dir. Giovanni Dotoli. Fasano : Schena, 2005. 11-28.

Morency, Jean. «La géographie des romans récents de France Daigle : un nouveau rapport à l'identité et à l'altérité ». Études canadiennes/Canadian Studies 58 (2005) : 195-203.

Boudreau, Raoul. «L'humour en mode mineur dans les romans de France Daigle ». Itinéraires et contacts de cultures 36 (2006) : 125-142.

Doyon-Gosselin, Benoit. «La figure de la maison et de l'architecte dans l'œuvre romanesque de France Daigle ». Port-Acadie 8-9 (2005-2006) : 61-74.

Francis, Cécilia W. «France Daigle. À propos des jeux de l'art et du hasard ». Canadian Literature 189 (2006) : 183-192.

Roy, Véronique. "La beauté de l'affaire : affaires d'architectes-écrivains ». Architecture, littérature et espaces. Dir. Pierre Hyppolite. Limoges : PU de Limoges, 2006. 29-38.

Boehringer, Monika. «"A Private Open Space" : Crossing Boundaries and Constructing Identities in France Daigle's Auto/Fictions ». Zeitschrift für Kanada-Studien 27.2 (2007) : 30-42.

Doyon-Gosselin, Benoit. «Le déplacement des références identitaires et des balises territoriales dans Variations en $B$ et en $K$ de France Daigle ». Balises et références. Acadies, francophonies. Dir. Martin Pâquet et Stéphane Savard. Québec : PU Laval, 2007. 371-393.

Gauvin, Lise. «Le lecteur et ses doubles : Dany Laferrière, France Daigle ». Écrire pour qui ? L'écrivain francophone et ses publics. Paris : Karthala, 2007. 127-144.

Gauvin, Lise. «Petites difficultés d'existence : la relation écrivain-lecteur dans les romans de France Daigle ». Québec Studies 43 (2007) : 23-28.

Giroux, François. « Agoraphobie et identité : mouvements d'affirmation inachevée dans Pas pire de France Daigle ». L'œuvre littéraire et ses inachèvements. Dir. Janine Gallant, Hélène Destrempes et Jean Morency. Longueuil : Groupéditions, 2007. 249-258.

Lavoie, Carlo. «Just Fine: The Bridge to the Non-Space from the Petitcodiac to the Deltas ». From One Shore to Another : Reflections on the Symbolism of the Bridge. Dir. Sanda Badescu. Newcastle, UK : Cambridge Scholars, 2007. 129-139.

Morency, Jean. « Perdus dans l'espace-temps : figures spatio-temporelles et inconscient diasporal dans les romans de France Daigle, Jean Babineau, Daniel Poliquin et Nicolas Dickner ». Balises et références. Acadies, francophonies. Dir. Martin Pâquet et Stéphane Savard. Québec : PU Laval, 2007. 487-509.

Paré, François. «Un fin passage de France Daigle ». À la carte. Le roman québécois (2000-2005). Dir. Gilles Dupuis et Klaus-Dieter Ertler. Frankfurt am Main : Lang, 2007. 107-121. 
Richard, Chantal. «La langue inachevée dans les romans de Jean Babineau et France Daigle ». L'œuvre littéraire et ses inachèvements. Dir. Janine Gallant, Hélène Destrempes et Jean Morency. Longueuil : Groupéditions, 2007. 239-248.

Boehringer, Monika. «Écrire ses racines. L'avant-texte de Sans jamais parler du vent de France Daigle : Fragments d'histoire et questions d'identité ». Lire du fragment: Analyses et procédés littéraires. Dir. Carlo Lavoie. Montréal : Nota Bene, 2008. 137-152.

Dumontet, Danielle. «Pour une poétique de la fragmentation à l'exemple de France Daigle ». Lire du fragment: Analyses et procédés littéraires. Dir. Carlo Lavoie. Montréal : Nota Bene, 2008. 355-370.

Leclerc, Catherine. "Le chiac, le Yi-King et l'entrecroisement des marges : Petites difficultés d'existence en traduction ». Traduire depuis les marges/Translating from the Margins. Dir. Denise Merkle, Jane Koustas, Glen Nichols et Sherry Simon. Québec : Nota Bene, 2008. 163-192.

Den Toonder, Jeannette. « France Daigle : voyage, espace et imaginaire ». Paysages imaginaires d'Acadie : un atlas littéraire. Dir. Marie-Linda Lord et Denis Bourque. Moncton : Institut d'études acadiennes, 2009 : 95-107.

Boehringer, Monika. «Au seuil du texte daiglien, la couverture : simple illustration ou porteuse de sens ? ». Constructions identitaires en Acadie et au Québec. Dir. Monika Boehringer, Kirsty Bell et Hans R. Runte. Moncton : Institut d'études acadiennes, 2010. 221-235.

Den Toonder, Jeannette. «L'acte créateur et l'espace littéraire dans l'autofiction de France Daigle (La beauté de l'affaire, 1953 et Pas pire) ». Relief 3.1 (2010) : 77-94.

Potvin, Claudine. "L'épaisseur de l'art: art et écriture chez France Daigle ». Entre textes et images. Constructions identitaires en Acadie et au Québec. Dir. Monika Boehringer, Kirsty Bell et Hans R. Runte. Moncton : Institut d'études acadiennes, 2010. 207-220.

Richard, Chantal. «Des mots comme les murs d'une maison : le leitmotiv du logis dans le roman acadien contemporain ». Studies in Canadian Literature/Études en littérature canadienne 35.1 (2010) : 165-179.

Ziethen, Antje. «France Daigles Puzzleroman : Spekularität und Fragmentierung in Pas pire ». Zeitschrift für Kanada-Studien 30.1 (2010): 101-112.

Doyon-Gosselin, Benoit. "Pour une herméneutique des espaces fictionnels ». Topographies romanesques. Dir. Audrey Camus et Rachel Bouvet. Rennes/Québec: PU de Rennes/PU du Québec, 2011.65-78.

Boehringer, Monika. «L'édition critique de Sans jamais parler du vent de France Daigle ». Port Acadie 20-21 (2011-2012) : 167-182. 
Leclerc, Catherine et Lianne Moyes. «Littérature et contiguïté. France Daigle au Québec, France Daigle et le Québec ». Voix et images 111 (2012) : 127-143.

Boudreau, Raoul. «Une réécriture ambiguë en littérature acadienne : Marguerite Duras et France Daigle ». Littératures francophones : Parodies, pastiches, réécritures. Dir. Lise Gauvin, Cécile Van den Avenne, Véronique Corinus et Ching Selao. Lyon : ENS Éditions, 2013. 91-104.

Bruyère, Vincent. «Études littéraires et écologie du minoritaire ». Francophonies d'Amérique 36 (2013) : 97-111.

Doyon-Gosselin, Benoit. « Le tournant spatio-référentiel dans l'œuvre romanesque de France Daigle ». Écrire au féminin au Canada français. Dir. Johanne Melançon. Sudbury : Prise de parole, 2013. 65-83.

Boehringer, Monika. «Les mots pour se/le dire : trois temps forts dans l'Acadie au féminin : Antonine Maillet, Dyane Léger, France Daigle ». Francophonies d'Amérique 37 (2014) : 173-201.

Viau, Robert. «Deltas erratiques. Pas pire de France Daigle». Acadie multipiste. Romans acadiens. Tome 1. Moncton : Perce-Neige, 2015. 155-184.

Dumontet, Danielle. «La fragmentation dans Pour sûr de France Daigle. Une écriture entre contraintes et ouvertures ». Littérature acadienne du 2le siècle. Dir. Cécilia W. Francis et Robert Viau. Moncton : Perce-Neige, 2016. 109-130.

Hotte, Lucie, «Au-delà de l'exiguïté : les œuvres de France Daigle, de André Christensen et de Simone Chaput ». Au-delà de l'exiguïté. Échos et convergences dans les littératures minoritaires. Dir. Jimmy Thibeault et al. Moncton : Perce-Neige, 2016. 31-51.

Papillon, Joëlle. «Regards croisés France-Acadie dans l'œuvre de France Daigle : norme, variation et expérimentations ». Littérature acadienne du 21e siècle. Dir. Cécilia W. Francis et Robert Viau. Moncton : Perce-Neige, 2016. 89-107.

Raymond, Maurice. «Pas pire de France Daigle ou le monde de la résonance et de l'ambiguïté ». Littérature acadienne du 21e siècle. Dir. Cécilia W. Francis et Robert Viau. Moncton : PerceNeige, 2016. 75-88.

Brun del Re, Ariane. «France Daigle, héritière de Gérald Leblanc : de Moncton mantra à Petites difficultés d'existence ». Revue de l'Université de Moncton 47.2 (2016) : 47-71.

Skidds, Catherine. «Réconcilier "espace" et "humain" : une lecture géocritique du roman Pas pire de France Daigle ». Littérature acadienne du 21e siècle. Dir. Cécilia W. Francis et Robert Viau. Moncton : Perce-Neige, 2016. 59-74.

Tremblay, Emmanuelle. «S'arracher à soi-même : les figures de la violence fondatrice du récit de soi chez Herménégilde Chiasson et France Daigle ». Les littératures franco-canadiennes à l'épreuve du temps. Dir. Lucie Hotte et François Paré. Ottawa : PU Ottawa, 2016. 231-255. 
Cabajsky, Andrea. «Francophone Acadian Literature as an Ultraminor Literature: The Case of Novelist France Daigle ». Journal of World Literature 2.2 (été 2017) : 158-177.

Doyon-Gosselin, Benoit. «De la maison à la métalepse daiglienne ». @ nalyses. Revue de critique et de théorie littéraire 12.3 (2017) : 75-100. https://uottawa.scholarsportal.info/ojs/index.php/revueanalyses/issue/view/281. Consulté le 17 janvier 2018.

Francis, Cecilia W. "Transgression et transmission dans Pour sûr de France Daigle. Entre éclatement formel et héritage linguistique ». Transmissions et Transgressions dans les littératures de l'Amérique francophone. Dir. Cecilia W. Francis et Robert Viau Moncton : Perce-Neige, 2017. 35-63.

\section{IV.2. Mémoires et thèses}

Lefrancois, Émilie. «Le désir d'“écrire au neutre”. Lecture des manifestations du genre dans les deux trilogies de France Daigle ». Mémoire de maîtrise. Rimouski : U du Québec à Rimouski, 2005.

Giroux, François. «La figure de l'écrivain chez France Daigle. Caractéristiques du discours ». Mémoire de maîtrise. Moncton : U de Moncton, 2006.

Doyon-Gosselin, Benoit. " Pour une herméneutique de l'espace : l'œuvre romanesque de J.R. Léveillé et France Daigle ». Thèse de doctorat. Moncton : U de Moncton, 2008.

Mallet, Janie. «D'un genre l'autre : l'adaptation de Sans jamais parler du vent de France Daigle ». Mémoire de maîtrise. Moncton : U de Moncton, 2013.

Brun del Re, Ariane. «Portraits de villes littéraires : Moncton et Ottawa ». Mémoire de maîtrise. Montréal : U McGill, 2013.

Cormier, Pénélope. "Écritures de la contrainte en littérature acadienne. France Daigle et Herménégilde Chiasson ». Thèse de doctorat. Montréal : U McGill, 2014.

Spencern, Trerice. «Entre fierté et mépris : le rapport ambivalent à l'égard du chiac dans Pour sûr de France Daigle ». Mémoire de maitrise. Victoria : U de Victoria, 2014.

Cormier, Matthew. "Ulyssean Traces in Postmodern Canadian Epics : Timothy Findley's The Wars and France Daigle's Pour sûr ». Mémoire de maîtrise. Moncton : U de Moncton, 2015.

Robidoux-Daigneault, Camille. «De Pas pire à Pour sûr : Faits et effets des langues chez France Daigle ». Mémoire de maitrise, Montréal : U de Montréal, 2016. 
Thivierge, Jessica. « Espaces et langues (dé)cloisonnés dans les quatre derniers romans de France Daigle ». Mémoire de maîtrise, Montréal : U McGill, 2016.

\section{IV.3. Entretiens et portraits}

Boehringer, Monika. «Le hasard fait bien les choses. Entretien avec France Daigle ». Voix et Images 87 (2004) : 13-23.

Savoie, Paul. Actes de création : entretiens. Ottawa : L’Interligne, 2006. 17-23.

Lonergan, David. «France Daigle ». Nuit Blanche 122 (2011) : 10-13.

Tardif, Dominic. «France Daigle: le poids des mots». Le libraire 74 (2012). http://revue.leslibraires.ca/entrevues/litterature-quebecoise/france-daigle-le-poids-des-mots

Consulté le 17 janvier 2018.

Barlow, Julie. «France Daigle : “Une langue, ça s’entretient” ». L'actualité (2013) : 60.

Doyon-Gosselin, Benoit. «France Daigle ». Dictionnaire des écrivains francophones classiques. Belgique, Canada, Québec, Luxembourg, Suisse romande. Dir. Corinne Blanchaud. Paris : Champion, 2013. 143-144.

Porcher, Claire. «La sculpture littéraire de France Daigle ». L'express, semaine du 10 au 16 décembre 2013. https://l-express.ca/la-sculpture-litteraire-de-france-daigle/. Consulté le 17 janvier 2018.

Cabajsky, Andrea. «"Le sentiment vif de créer" : entretien avec France Daigle/“The Vivid Feeling of Creating" : An Interview with France Daigle ». Studies in Canadian Literature / Études en littérature canadienne 39.2 (2014) : 248-269.

Grenier, Daniel. «Daniel Grenier à France Daigle ». Moebius : écritures/littérature 141 (2014) : 139-147.

Boehringer, Monika. «France Daigle et son Acadie, ouverte sur le monde ». Encyclopédie du patrimoine culturel de l'Amérique française.

http://www.ameriquefrancaise.org/fr/article

595/France_Daigle_et_son_Acadie,_ouverte_sur_le_monde.html\#.WLWZ3FJTHcs. Consulté le 17 janvier 2018.

Bourque, Denis. « France Daigle ». L’Encyclopédie canadienne. 2015. http://www.encyclopediecanadienne.ca/fr/article/france-daigle/. Consulté le 17 janvier 2018. 


\section{IV.4. Comptes rendus et articles de presse \\ IV.4.1 Sans jamais parler du vent}

Décarie, David. «Sans jamais parler du vent ». Dictionnaire des ouvres littéraires de l'Acadie des maritimes. Dir. Janine Gallant et Maurice Raymond. Sudbury : Prise de parole, 2012. 246-248.

Nicaise, Nicolas. «Sans jamais parler du vent. Roman de crainte et d'espoir que la mort arrive à temps. Éd. critique établie par Monika Boehringer ». Francophonies d'Amérique 35 (2013) : 176179.

\section{IV.4.2 Film d'amour et de dépendance}

Doyon-Gosselin, Benoit. «Film d'amour et de dépendance ». Dictionnaire des æuvres littéraires de l'Acadie des maritimes. Dir. Janine Gallant et Maurice Raymond. Sudbury : Prise de parole, 2012. 129-130.

\section{IV.4.3 Histoire de la maison qui brûle}

Boehringer, Monika. «Histoire de la maison qui brûle » [Compte rendu de la pièce de MonctonSable d'après le texte de France Daigle]. Liaison 139 (2008) : 42.

Dumontet, Danielle. «Histoire de la maison qui brûle ». Dictionnaire des ceuvres littéraires de l'Acadie des maritimes. Dir. Janine Gallant et Maurice Raymond. Sudbury : Prise de parole, 2012. 147-148.

\section{IV.4.4 Variations en $B$ et $K$}

Doyon-Gosselin, Benoit. «Variations en B et $K$ ». Dictionnaire des auvres littéraires de l'Acadie des maritimes. Dir. Janine Gallant et Maurice Raymond. Sudbury : Prise de parole, 2012. 275-276.

\section{IV.4.5 L'été avant la mort}

Giroux, François. «L'été avant la mort ». Dictionnaire des æeuvres littéraires de l'Acadie des maritimes. Dir. Janine Gallant et Maurice Raymond. Sudbury : Prise de parole, 2012. 116-117.

\section{IV.4.6 La beauté de l'affaire}

Doyon-Gosselin, Benoit. «La beauté de l'affaire ». Dictionnaire des œuvres littéraires de l'Acadie des maritimes. Dir. Janine Gallant et Maurice Raymond. Sudbury : Prise de parole, 2012, 23-24. 


\section{IV.4.7 La vraie vie}

Dumontet, Danielle. "La vraie vie ». Dictionnaire des cuvres littéraires de l'Acadie des maritimes. Dir. Janine Gallant et Maurice Raymond. Sudbury : Prise de parole, 2012. 287-290.

\section{IV.4.9 1953. Chronique d'une naissance annoncée}

Lonergan, David. «La magnifique histoire de bébé M. ». Tintamarre. Chroniques de littérature dans l'Acadie d'aujourd'hui [Reproduction d'une critique parue dans le quotidien L'Acadie nouvelle]. Sudbury : Prise de parole, 2008. 109-111.

Boehringer, Monika. «1953. Chronique d'une naissance annoncée ». Dictionnaire des æuvres littéraires de l'Acadie des maritimes. Dir. Janine Gallant et Maurice Raymond. Sudbury : Prise de parole, 2012. 1-2.

Landry, Pierre-Luc. «1953: le roman de tous les discours». Astheure. 10 juillet 2015. https://astheure.com/2015/07/10/1953-le-roman-de-tous-les-discours-pierre-luc-landry/. Consulté le 17 janvier 2018.

\section{IV.4.10 Pas pire}

Lonergan, David. «Un beau et doux voyage ». Tintamarre. Chroniques de littérature dans l'Acadie d'aujourd'hui [Reproduction d'une critique parue dans le quotidien L'Acadie nouvelle]. Sudbury : Prise de parole, 2008. 111-113.

Giroux, François. «Pas pire ». Dictionnaire des œuvres littéraires de l'Acadie des maritimes. Dir. Janine Gallant et Maurice Raymond. Sudbury : Prise de parole, 2012. 200-203.

\section{IV.4.11. Un fin passage}

Lonergan, David. «Un fin et délicat passage vers la vie ». Tintamarre. Chroniques de littérature dans l'Acadie d'aujourd'hui [Reproduction d'une critique parue dans le quotidien L'Acadie nouvelle]. Sudbury : Prise de parole, 2008. 113-116.

\section{IV.4.12 Petites difficultés d'existence}

Hudson, Jean-Guy. «Petites difficultés d'existence ». Nuit blanche 91 (2003) : 19-20. 
Lonergan, David. «France Daigle : un univers de lumière ». Tintamarre. Chroniques de littérature dans l'Acadie d'aujourd'hui [Reproduction d'une critique parue dans le quotidien L'Acadie nouvelle]. Sudbury : Prise de parole, 2008. 116-118.

\section{IV.4.13 Pour sûr}

Fortin, Marie-Claude. «Pour sûr de France Daigle : l'œuvre ouverte ». La Presse 16 (septembre 2011).

Doyon-Gosselin, Benoit. « 145. Le chef-d'œuvre de la contrainte ». Liaison 154 (2011-2012) : 6263.

Tardif, Dominic. «Pour sûr ». Voir, 26 janvier 2012 https://voir.ca/livres/2012/01/26/france-daigle-pour-sur-2/. Consulté le 17 janvier 2018.

Grenier, Daniel. « Lecture angulaire de Pour sûr, le dernier roman de France Daigle ». Blog StHenri, 2 février 2012.

https://sthenri.wordpress.com/2012/02/02/lecture-angulaire-de-pour-sur-le-dernier-roman-defrance-daigle-i/. Consulté le 17 janvier 2018.

Brochu, André. « Jacques Poulin, France Daigle ». Lettres québécoises 145 (2012) : 18-19.

Brun del Re, Ariane. «Pour sûr par France Daigle ». Nouvelles études francophones 27.2 (2012) : 251-254.

Ferland, Pierre-Paul. «Ceci n'est pas un roman sur le chiac ». Salon double 8 (2012)

http://salondouble.contemporain.info/ceci-n-est-pas-un-roman-sur-le-chiac_. Consulté le 17 janvier 2018.

Lefort-Favreau, Julien. « Chiac, langue première, langue littéraire ». Liberté 54.2 (2013) : 30-31.

Parayre, Catherine. «Pour sûr ». Voix plurielles 11.1 (2014)

https://brock.scholarsportal.info/journals/voixplurielles/article/view/941. Consulté le 1er décembre 2017.

\section{IV.4.14 Poèmes pour vieux couples}

Nicaise, Nicolas. «Une écriture aux ciseaux ». Liaison 175 (2017) : 57.

Bouchard, Geneviève. «Poèmes pour vieux couples, un recueil inabouti ». Astheure. 29 mai 2017. https://astheure.com/2017/05/29/poemes-pour-vieux-couples-un-recueil-inabouti-genevievebouchard/. Consulté le 17 janvier 2018. 